Volume. 5 Number. 2

Period: July - December 2021; page 62-68

p-ISSN : 2580-1112; e-ISSN : 2655-6669

Copyrighr@2020

The author owns the copyright of this article

journal homepage: https://ejournal.akperfatmawati.ac.id

DOI : 10.46749/iiko.v5i2.73
Jurnal IImiah Keperawatan

Orthopedi (JIKO)

Article history:

Received: September 16, 2021

Revised: February 11, 2022

Accepted: February 13, 2022

\title{
The Position of Leaning Forward to Reduce Breathing of COPD Patients: Literature Review
}

\author{
Aat Djanatunisah ${ }^{1}$, Riri Maria ${ }^{2}$ \\ ${ }^{1}$ Master of Nursing Student Specialization in Medical Surgery, FIK UI, ${ }^{2}$ FIK UI lecturers \\ Fatmawati Hospital, Jl. RS. Fatmawati, Cilandak, South Jakarta 12430 \\ E-mail: djanatunisah@gmail.com
}

\begin{abstract}
COPD patients often experience shortness of breath as a result of inflammation in the lungs that develops in the long term. COPD is generally characterized by difficulty breathing, coughing with phlegm, and wheezing or wheezing. In this condition, the patient often finds a comfortable position to reduce shortness of breath. The aim of this study was to look at appropriate and effective interventions in reducing spasms in COPD patients. This literature study was created by analyzing scientific articles, at least in retrospective research from 2012 to 2020 and in English. Data obtained from databases include Pubmed, CINAHL, Scopus, Proquest and Google Scholar with the keywords position for breathing in COPD, position forward lean trunk for short breathing in COPD, and best position for COPD. The results of this literature obtained 5 articles that match the inclusion and exclusion criteria. The results have been found that the forward leaning position is effective in reducing shortness of breath in COPD patients because of the involvement of the respiratory accessory muscles so that the pulmonary ventilation process is good.
\end{abstract}

Keywords: Position for breathing, forward leaning position, COPD

\section{Background}

Chronic Obstructive Pulmonary Disease (COPD) is a preventable and treatable disease characterized by progressive limited airflow associated with chronic inflammatory response to the airways and lungs due to toxic particles or gases (Disease Global Initiative For Chronic Obstructive Lung. 2017). The incidence of COPD has reached more than 5 percent of the total population and is associated with increased morbidity and mortality. In America it has become the third leading cause of death with a death rate of more than 120,000 people each year (Miniño et.al., 2011).
In 2020, it is estimated that COPD will become the third cause of death in the world after ischemic heart disease and cerebrovascular disease (Angelis. Et. Al., 2014). Based on the 2013 Basic Health Research (RISKESDAS) data in Indonesia, the prevalence of COPD is $3.7 \%$.

The incidence of this disease increases with increasing age and is higher in men $(4.2 \%)$ than in women $(3.3 \%)$ (Riskesdes, 2013). In COPD, exacerbations and comorbids contribute to the overall severity of the disease in a patient. (Disease Global Initiative For Chronic Obstructive Lung. 2017). 
COPD can cause interference with the oxygenation process of the entire body due to alveolar damage and changes in respiratory physiology. These damage and changes can cause inflammation of the bronchi and result in damage to the walls of the terminal bronchioles and cause obstruction or early closure of the expiratory phase resulting in airway limitations that are not completely reversible due to the inflammatory response. (Singh. Et.al., 2019)

Chronic airflow inhibition in COPD is a combination of small airway disease and parenchymal destruction with differing patient-to-patient contributions. In fact, COPD is a group of diseases with clinical symptoms similar to those of chronic bronchitis, emphysema, asthma, bronchiectasis, and bronchiolitis. Airway obstruction that occurs in patients with COPD is caused by diseases of the airways and damage to the lung parenchyma. (Stockley. Et. Al., 2009).

According to Chronic Obstructive Pulmonary Disease (COPD) International (2012), patients with Chronic Obstructive Pulmonary Disease (COPD) experience changes in the shape of the chest. The deformation that occurs is the proportional diameter of the anteroposterior and transverse chest shape, often called barrel chest. Difficulty breathing also occurs in patients with Chronic Obstructive Pulmonary Disease (COPD), which is breathing using a breathing muscle for a long time, then muscle hypertrophy and widening between the ribs or the intercostal area will occur.

Breathing support muscles are necessary for the lungs to expand properly during the ventilation process. Therefore, in order for the breathing muscles to work properly, a position is needed that can help develop the lungs. One of the nursing interventions given is to adjust the position of the patient leaning forward to reduce the tightness and maximize the work of the breathing muscles.

Leaning forward in COPD patients improves the pressure-length relationship and the geometry of the diaphragm, which improves the breathing process. (Sharp. Et. Al., 1980). Leaning forward also aims at contraction of the diaphragm to increase chest wall motion, thereby increasing lung volume changes (Delgado. Et. Al., 1982). Leaning forward with both arms and head can increase the activity of the inspiratory accessory muscles during inspiration in COPD. (Kim. Et. Al., 2012). Leaning forward with arm supports while standing or sitting increases the recruitment of the inspiratory accessory muscles namely the sternocleidomastoid (SCM) and scalenus (Sc). which increases the anterior-toposterior intercostal movement. (Montes. Et. Al., 2018).

Leaning forward will raise the diaphragm and external intercostal muscles to a position of approximately 45 degrees. The diaphragm muscles are the main muscles of inspiration and the external intercostal muscles are also the muscles of inspiration. The diaphragm muscle which is at a position of 45 degrees causes the earth's gravitational force to work quite adequately on the main muscle of inspiration compared to a sitting or half-sitting position.

The gravitational force of the earth acting on the diaphragm muscle makes it easier for the muscle to contract and move downward, increasing the volume of the thoracic cavity by increasing its vertical length. Likewise with the external intercostal muscles, the earth's gravitational force acting on the muscle makes it easier for the ribs to lift outward, thereby enlarging the thoracic cavity in the anteroposterior dimension.

\section{Materials and Methods}


Search for relevant articles using databases such as CINAHL, Pubmed, Google scholar, Scopus and Proquest. Search for articles using keywords: position for breathing, Forward leaning position, COPD. The articles taken were selected using inclusion and exclusion criteria including articles in English, published between 2012-2020, all COPD patients from degrees 0 -IV and healthy respondents and full text.

The author also uses several design studies from several studies obtained such as quasi experimental design, and cross sectional.

\section{Result}

Based on the search results by entering keywords obtained from Pubmed 2 articles, CINAHL 2 articles, Scopus 4 articles, Google scholar 375 and Proquest 9,629 articles. After sorting it was obtained from, Scopus 3 articles, Proquest 2 articles and Google scholar 2, a total of 11 articles.

However, only 7 articles have the structure of an article and relate to the position of the patient to reduce tightness in COPD patients. Furthermore, skimming and scanning were carried out, there were 4 articles issued on the grounds that the research did not meet the inclusion criteria.

\section{Discussion}

Based on the 7 articles analyzed, it consisted of a quasi-experimental study of 5 articles and 2 cross-sectional articles. Of the 7 articles, 6 articles assessed different positions to improve respiratory muscles and lung function, while 1 article assessed forward leaning combined with physiotherapy or breathing exercises.

The results of the 7 articles state that leaning forward can increase the activity of the respiratory muscles and breathing accessory muscles thereby helping to reduce shortness of breath due to weakness of the respiratory muscles that occurs in COPD patients. Several studies on position are also combined with physiotherapy which aims to increase lung capacity volume, inspiratory and expiratory respiratory function and to clear the airway of secretions in COPD patients. From the research results obtained, an assessment of standardized measuring instruments such as EMG, spirometry, microRPM, qualisys motion capture system, inductive respiratory plethysmography, oximetry and OEP has been carried out so that the assessment is more accurate.

This research also exists as a followup to previous studies conducted by several researchers so that the results are more relevant and effective. The results of the research analyzed stated that the forward leaning position of the diaphragm muscle and external intercostal muscles experienced an increase in activity which caused the earth's gravity to work quite adequately on the inspiratory main muscles compared to sitting or half-sitting positions. (Booth., 2006). The earth's gravitational force acting on the diaphragm muscle makes it easier for these muscles to contract moving downward, increasing the volume of the thoracic cavity by increasing the vertical length of the chest cavity, as well as the external intercostal muscles, the earth's gravitational force acting on these muscles makes it easier for the ribs to be lifted out so that it enlarges the thoracic cavity. in the anteroposterior dimension (Khasanah. 2013).

The enlarged thoracic cavity causes the pressure in the thoracic cavity to expand and forces the lungs to expand, thereby decreasing the intra-alveolar pressure. The drop in intra-alveolar pressure is lower than the atmospheric pressure causing air to flow into the pleura. This process suggests that leaning 
forward makes it easier for patients with shortness of breath who experience an obstructive airway to inspire without expending too much energy. The process of inspiration using less energy can reduce patient fatigue while breathing and also minimize oxygen use.

The forward leaning position increases intraabdominal pressure and decreases diaphragmatic pressure to the abdominal cavity during inspiration (Bhatt, et al, 2009). The intra-abdominal muscles are the main muscles for expiration. Increased intra-abdominal muscle contraction will increase intra-abdominal pressure.

The increase in intra-abdominal pressure will push the diaphragm upward, lifting the thoracic cavity, thereby reducing the size of the thoracic cavity. Another expiratory muscle, the internal intercostal muscle, is positioned forward leaning the muscle at an angle of about 30-45 degrees, which allows the gravitational force to work more optimally. The earth's gravity will help pull the internal intercostal muscles downward so that the size of the thoracic cavity becomes smaller. The smaller the size of the thoracic cavity makes the intra alveolar pressure increase. An increase in intra alveolar pressure that exceeds atmospheric pressure causes air to flow out of the lungs. The increased ventilation process in patients with shortness of breath who are positioned leaning forward will increase $\mathrm{CO} 2$ output and increase oxygen intake into the intra alveoli (Bhatt. Et.al. 2009)

This study emphasizes the importance of giving a forward leaning position in COPD patients in order to reduce shortness of breath that occurs. Based on the analysis in giving forward leaning positions, there are things that support the success of this intervention, including differences in the degree of tilt in the forward leaning position, the respiratory muscles involved in the forward leaning position and the combination of forward leaning with physiotherapy or breathing exercises.

\section{The difference in the angle of the forward leaning position}

The difference in the degree of tilt in the forward leaning position can affect lung function such as lung volume, IRV, ERV and the use of the breathing muscles to improve pulmonary ventilation function. Whereas the tidal volume has no difference with the forward leaning position with different degrees (Lee et al. 2017)

In Lee's research. et.al.2017 also stated that a 30 degree forward leaning position is most effective at improving lung function because using gravity helps to expand the chest and relieves pressure on the abdomen and diaphragm. According to Khasanah 2015, leaning forward will increase the diaphragm and external intercostal muscles to a position of approximately 45 degrees.

\section{The main muscles of the breathing and the auxiliary muscles are involved in the forward leaning position}

The forward leaning position increases intraabdominal muscle pressure and decreases the pressure of the diaphragm muscle to the abdominal cavity during inspiration (Bhatt, et al, 2009). In a study conducted by Kim, et al (2012), the activity of the Scalene and Sternocledomastoideus muscles significantly increased in forward leaning positions with arms supported on the thighs or arms supported by the head compared to neutral positions.

Leaning forward with a shoulder support (pectoralis major and minor muscles) contributes significantly to the development of the ribs or intercostals (Gosselink, 2013). According to Montes. et.al. 2017 forward leaning and 4 point 
kneeling positions can increase Transversus Abdominis / Internal Oblique ( $\operatorname{TrA} / \mathrm{IO})$ during the process of breathing.

\section{Forward leaning position and Respiratory physiotherapy}

Physiotherapy or breathing exercises are useful for improving chest wall movement to reduce the work of breathing, muscle accesories and dyspnea as well as to improve breathing efficiency and improve ventilation distribution (KNGF. 2008). Physiotherapy as a form of non-pharmacological health service can help sufferers of chronic obstructive pulmonary disease to recover physically and improve their breathing patterns so that they can break the chain of complaints that become mutual causes and effects. Leaning forward in combination with physiotherapy is very helpful for clearing the airway and improving pulmonary ventilation in COPD patients.

Purse Lip breathingand the Active Cycle of Breathing Technique are examples of physiotherapy given to COPD patients. Pursed Lip Breathing is a pulmonary rehabilitation that is very easy to do without using assistive devices and there are no side effects that aim to improve oxygen transport, induce slow and deep breathing patterns, control breathing, prevent collapse, and train the expiratory muscles to prolong exhalation and increase airway pressure during expiration and reduce the amount of trapped air or air trapping (Smeltzer \& Bare, 2013).

PLB is a breathing exercise that emphasizes the expiration process which is done calmly and relaxed with the aim of facilitating the process of expelling air trapped by the airways. Through this technique, the air that comes out will be blocked by the lips, which causes the pressure in the oral cavity to be more positive. This positive pressure will spread into the narrowed airway and is useful for keeping the airway open. With the opening of the airway, air can easily escape through the narrowed airway and easily affects the strength of the respiratory muscles to reduce shortness of breath (Permadi, \& Wahyudi, 2017).

Pursed Lip Breathing or breathing exercise is done by breathing or inspiring through the nose for 2-3 seconds followed by slow expiration through the mouth at least 2 inspirations (4-6 seconds) carried out for 30 minutes with a resting pause tolerance of 5 minutes for 3 times ( 5 interventions, a 5 minute break, continued 5 minutes to 2 and a 5 minute break, Active Cycle of Breathing Technique(ACBT) is a physiotherapy chest that aims to clear the airway in patients with Chronic Obstructive Pulmonary Disease (NHS, 2009).

ACBT also aims to reduce shortness of breath, better control breathing and management of breathlessness as well as better mobilization or movement of the thoracic cavity. ACBT consists of three sub-techniques, namely Breathing Control (BC), Thoracic expansion exercise (TEE) and forced expiration technique (FET) which can be done together or one by one. Breathing exercises increase the inspiratory capacity and stimulate the work of the respiratory muscles.

Huffing exercises increase the tidal volume and open the collateral system of the airways so that the spitum is easily expelled. Breathing Control aims to reeducate calm and regular breathing patterns so that patients can save energy for breathing and patients will get used to doing regular breathing when attacks of shortness of breath. The combination of the two subtechnics can be done together with thoracic cavity mobility exercises and postural improvement. 


\section{Conclusion}

The forward leaning position is very effective and easy to perform as a nursing intervention to reduce shortness of breath in COPD patients. Leaning forward can increase the activity of the respiratory muscles and breathing accessory muscles, increasing the volume of the lung capacity so that the lungs can expand maximally and this has been proven by several studies conducted.

The forward leaning position can also be combined or combined with other forms of physiotherapy or breathing exercises as needed to treat respiratory problems and in COPD patients. Therefore, this intervention can be applied in health services as a nonpharmacological therapy in dealing with shortness of breath.

The LOE 7 of this article is Level IV, so more complex and advanced evidence is needed, however, this study can represent a tested and proven nursing intervention that can be administered to COPD patients with breathing problems to reduce shortness of breath.

\section{Reference}

Albarrati, Ali., Hamayun Zafar., Alghadir., Ahmad H., Shahnwaz Anwer., (2018). Effect of Upright and Slouched Sitting Postures on the Respiratory Muscle Strength in Healthy Young Males. National Library of Medecine. DOI: 10.1155 / 2018/3058970

Angelis, N., Porpodis, K., Zarogoulidis, P., Spyratos, D., Kioumis, I., Papaiwannou, A., Pitsiou, G. et al. (2014). Airway inflammation in chronic obstructive pulmonary disease. J. Thorac. Dis. 6, 49.

António Mesquita Montesa., Carolina Tam., Carlos Crastoa., Cristina Argel de Meloa., Paulo Carvalhoa., Rita Santosa., Rui Vilarinhoa., João Paulo Vilas-Boas. (2018). Forward trunk lean with arm support affects the activity of accessory respiratory muscles and thoracoabdominal movement in healthy individuals. Elsevier. Human Movement Science 61.167-176

António Mesquita Montesa., Carolina Tam., Carlos Crastoa., Cristina Argel de Meloa., Paulo Carvalhoa., Rita Santosa., Rui Vilarinhoa., João Paulo Vilas-Boas. (2017). Abdominal muscle activity during breathing in different postures in COPD "Stage 0" and healthy subjects. Elsevier. Respiratory Physiology \& Neurobiology 238. 14-22

Bhatt, SP., Guleria, R., Luqman-Arafath, TK., Gupta, AK., Mohan, A., Nanda, S., \& Stoltzfus, JC 2009. Effect of tripod position on objective parameters of respiratory function in stable chronic obstructive pulmonary disease. Indian J Chest Dis Allied Sci. 51: $83-85$

Booth., (2006). APARQ (Adolscent Physical Activity Recall Questionare. University Sydney: NSW.

Delgado, HR, Braun, S R., Skatrud, JB, Reddan, WG, \& Pegelow, DF (1982). Chest wall and abdominal motion during exercise in patient with chronic obstructive pulmonary disease. The American Revieum of Pulmonary Disease, 126, 200-2005

Disease Global Initiative For Chronic Obstructive Lung. Pocket Guide To Copd Diagnosis, Management, And Prevention A Guide for Health Care Professionals 2017 EDITION.

Gosselink, 2013.Controlled Breathing and Dyspnea in Patients with Chronic Obstructive Pulmonary Disease (COPD). Journal of Rehabilitation Research and Development. Vol. 40 No.5. 
Indonesia KKR. (2013). Basic Health Research 2013.

Juncheol Lee., And Dongwook Han. (2017). Effect of the trunk forward bending angle in sitting position on slow vital capacity. Physical Therapy Science. 12: 2220-2223.

Khasanah, S., \& Maryoto, M. (2016). Effect of leaning forward (CKD) with pursed lips breathing (PLB) on the improvement of the respiratory conditions of patients with Chronic Obstructive Pulmonary Disease (COPD)

Ki-song Kim., Min-kwang Byun., Wonhwee Lee., Heon-seock Cynn., Ohyun Kwon., And Chung-hwi Yi. (2012). Effects of breathing maneuver and sitting posture on muscle activity in inspiratory accessory muscles in patients with chronic obstructive pulmonary disease. Multidisciplinary Respiratory Medicine

KNGF. (2008). Chronic Obstructive Pulmonary Disease. Practice Guidelines. England: Royal Dutch Society For Physical Therapy.

Miniño., BAM., Murphy, SL \& Xu, J. (2011). National Vital Statistics Reports Deaths: Final Data for 2008. 59.

NHS. (2009). National Health Service Standard Contract. NHS England. Pp. 1-22. Available at: england.nhs.uk.

Nutsupa Ubolnuar., Anong Tantisuwat., Premtip Thaveeratitham., Somrat Lertmaharit., Chathipat Kruapanich., Jaturong Chimpalee., And Witaya Mathiyakom. (2020). Effects of pursed-lip breathing and forward trunk lean postures on total and compartmental lung volumes and ventilation in patients with mild to moderate chronic obstructive pulmonary disease. Medicine: doi: 10.1097 / MD.0000000000023646

Permadi, AW., \& Wahyudi, AT. (2017). The effect of pursed lip breathing and sustained maximal inspiration to increase the strength of the respiratory muscles to reduce complaints of shortness of breath in cases of cardio respiration. Interest: Journal of Health Sciences, 6 (2)

Singh D., Agusti A., Anzueto A., Barnes PJ., Bourbeau J., Celli BR, et al. (2019). Global Strategy for the Diagnosis, Management, and Prevention of Chronic Obstructive Lung Disease: the GOLD science committee report 2019. The European respiratory journal; 53 (5).

Sharp, JT, Drutz, WS, Moisan, T., Foster, J., \& Machnach, W. (1980). Postural relief of dyspnoe in severe chronic obstetrical pulmonary disease. The American Review of Respitaory disease, 122.201-211.

Stockley RA., Mannino D., Barnes PJ. (2009). Burden and Pathogenesis of Chronic Obstructive Pulmonary Disease. Proceedings of the American Thoracic Society; 6 (6): 524-6.

Zuriati Zuriatia., Melti Suryab., Zahlima. (2020). Effectiveness Active Cycle of Breathing Technique (ACBT) with Pursed Lips Breathing Technique (PLBT) to tripod position in increase of oxygen saturation in patients with COPD, West Sumatera. Elsevier. Enferimeria Clinica. 164-167. 